\title{
Abordagem racional no planejamento de novos tuberculostáticos: Inibidores da InhA, enoil-ACP redutase do M. tuberculosis
}

\author{
Carolina Horta Andrade*1, Kerly Fernanda Mesquita Pasqualoto², Márcio Henrique Zaim³ \\ Elizabeth Igne Ferreira ${ }^{1}$
}

'LAPEN, Departamento de Farmácia, Faculdade de Ciências Farmacêuticas, Universidade de São Paulo, 'Laboratório de Quimiometria Teórica e Aplicada, Departamento de Físico-Química, Instituto de Química, Universidade Estadual de Campinas, ${ }^{3}$ Sintefina Indústria e Comércio Ltda

*Correspondência:

C. H. Andrade

LAPEN - Departamento de Farmácia

Faculdade de Ciências Farmacêuticas

Universidade de São Paulo

Av. Prof. Lineu Prestes, 580, Bloco 13

Cidade Universitária,

05508-900 - São Paulo - SP, Brasil

E-mail: carolhorta@usp.br
Associada à disseminação da infecção causada pelo HIV, a tuberculose (TB) é considerada, atualmente, problema mundial de saúde pública devido às proporções que vem assumindo. A resistência micobacteriana aos fármacos utilizados na terapêtica é a principal causa da reincidência da TB. Diante deste quadro alarmante, o desenvolvimento de novos e seletivos fármacos anti$T B$ se faz urgente e necessário. A biossintese de ácidos graxos é um processo bioquímico realizado por procariotos e eucariotos, o qual fornece precursores essenciais à montagem de componentes celulares importantes, tais como fosfolipideos, lipoproteinas, lipopolissacarídeos, ácidos micólicos e envelope celular. As diferenças bioquímicas e funcionais entre o mecanismo biossintético de ácidos graxos em bactérias e mamiferos tornamno alvo relevante ao planejamento de novos antibacterianos, mais seletivos e menos tóxicos. As enoil-ACP redutases são enzimas cruciais à etapa de alongamento de ácidos graxos, considerados produtos intermediários na biossintese de ácidos micólicos - os principais componentes da parede celular micobacteriana. Portanto, tais enzimas são tidas como alvos moleculares no planejamento racional de novos tuberculostáticos. Avanços recentes no processo de descoberta de novos agentes anti-TB, particularmente os inibidores da enoil-ACP redutase, serão discutidos nesta revisão.
Unitermos

- Tuberculose

- Ácidos graxos/biossíntese

- Ácidos micólicos

- Resistência micobacteriana

- Fármacos/ planejamento com base na estrutura

- Enoil-ACP redutase/ inibidores

\section{TUBERCULOSE E EPIDEMIOLOGIA}

A tuberculose (TB) é a principal causa de mortalidade por uma única doença infecciosa e é responsável pela morte de mais de 2 milhões de pessoas em todo o mundo anualmente (WHO, 2007). A TB é considerada problema global de saúde pública e os principais fatores que implicaram esta situação são: (i) falta de adesão dos pacientes aos esquemas terapêuticos disponíveis, com duração de seis a nove meses; (ii) o aparecimento de cepas de TB multiresistentes (MDR-TB - "multidrug-resistant") (WHO, 2007; Singh, 2004; Gandy, Zumla, 2002), que são defini- 
das pela resistência aos fármacos isoniazida e rifampicina, e (iii) a co-infecção com o vírus HIV, em que a TB é a principal causa de morte entre os pacientes HIV-positivos (Espinal, 2003; Zhang, Post-Martens, Denkin, 2006).

Embora a vacina disponível para a TB, a BCG (Bacilo de Calmette-Guérin), previna o desenvolvimento de TB fatal em crianças e jovens, sua eficácia de proteção contra TB pulmonar em adultos é questionável. Nenhuma outra vacina eficaz para reduzir a incidência de TB em adultos encontra-se, atualmente, disponível (Nayyar, Jain, 2005). Portanto, a principal estratégia para o controle da disseminação da TB é a quimioterapia (Corbett et al., 2003; Zhang, 2005; Janin, 2007). Sendo assim, novos agentes anti-TB são urgentemente necessários para diminuir a incidência global da TB. A elucidação dos mecanismos de resistência do microrganismo aos fármacos disponíveis pode representar avanço significativo no desenvolvimento de novos agentes anti-TB capazes de agir contra cepas MDR-TB (Oliveira et al., 2006; Oliveira et al., 2007).

As infecções causadas pela micobactéria são, em geral, difíceis de tratar devido à sua resistência natural à maioria dos antibióticos e agentes quimioterápicos (Dolin et al., 1994; Jarlier, Nikaido, 1994). Tal resistência é provocada em grande parte pela constituição da parede celular micobacteriana, que funciona como uma barreira física a muitos fármacos. A parede celular é formada por componentes altamente lipofílicos e os principais são: (1) ácidos graxos de cadeia longa, denominados ácidos micólicos, e (2) polissacarídeos singulares, denominados arabinogalactanos (Brenan, Nikaido, 1995; Barry, Mdluli, 1996).

\section{BIOSSÍNTESE DE ÁCIDOS GRAXOS}

A biossíntese bacteriana de ácidos graxos tem despertado atenção especial como alvo atraente no desenvolvimento de novos agentes antibacterianos (Heath, White, Rock, 2001, 2002; Payne et al., 2002). Tal processo é essencial para todos os seres vivos, já que fornece precursores a componentes celulares importantes, incluindo fosfolipídeos, lipoproteínas, lipopolissacarídeos, ácidos micólicos e o envelope celular da micobactéria (Heath, White, Rock, 2001, 2002)

A disponibilidade em bancos de dados de estruturas tridimensionais, obtidas por cristalografia de raios- $X$, da maioria das enzimas envolvidas no processo bioquímico bacteriano/micobacteriano tem contribuído significativamente à elucidação tanto do mecanismo catalítico como das características estruturais relevantes envolvidas neste processo. Além disso, a disponibilidade de inibidores co-cristalizados com tais enzimas permite a melhor compreensão das interações enzima-inibidor, favorecendo sua otimização e/ou o planejamento de melhores inibidores (Marrachi, Zhang, Rock, 2002).

O mecanismo biossintético dos ácidos graxos, denominado sistema FAS ("Fatty Acid Synthase"), envolve ciclos repetitivos de condensação, ceto-redução, desidratação e enoil-redução. A maioria dos eucariotos, exceto as plantas, sintetiza os ácidos graxos pelo sistema FAS tipo I, no qual a atividade enzimática reside em uma única cadeia polipeptídica de uma enzima multifuncional. As bactérias, as plantas e o Plasmodium falciparum contêm um sistema FAS tipo II, no qual cada reação é catalisada por uma enzima diferente. Em ambos os casos, o substrato é continuamente ativado via ligação tioéster ao grupo prostético do cofator requerido, coenzima A, CoA, ou proteína acilcarregadora, ACP (Heath, White, Rock, 2001, 2002; Marrachi, Zhang, Rock, 2002).

Já as micobactérias possuem ambos os sistemas FAS I e II. Neste caso, o sistema FAS II utiliza os produtos do sistema FAS I como base para o alongamento mais uniforme da cadeia de ácido graxo (Brenan, Nikaido, 1995; Barry et al., 1998). Os produtos de cadeia mais longa são os precursores dos ácidos micólicos, principais componentes da parede celular micobacteriana (Barry et al., 1998).

Como já mencionado anteriormente, tais diferenças bioquímicas e funcionais fazem com que as enzimas envolvidas na biossíntese micobacteriana de lipídeos sejam alvos promissores para o planejamento de novos agentes antimicobacterianos mais potentes e seletivos (Levy et al., 2001).

Alguns inibidores conhecidos, naturais e sintéticos, têm sido descritos como antibacterianos/ antimicobacterianos em razão de inibir a biossíntese de ácidos graxos (Figura 1).

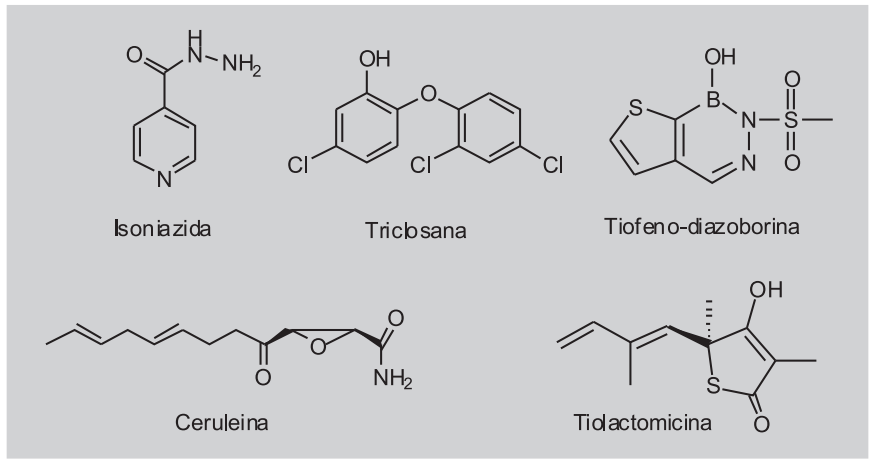

FIGURA 1 - Inibidores da biossíntese de ácidos graxos bacteriana.

\section{Sistema FAS II}

A via tipo II de biossíntese de ácidos graxos tem sido extensivamente estudada na Escherichia coli, devido ao seu maior seqüenciamento genômico e, também, em razão 
de as enzimas relacionadas nesta via serem altamente conservadas entre as espécies (Payne et al., 2002; Heath, White, Rock, 2001).

A primeira enzima envolvida no sistema FAS II é a acetil-CoA carboxilase, enzima multi-subunidades (AccABCD) (Figura 2). O produto da sua reação é a malonil-CoA. O grupo malonato é transferido à ACP pela malonil-CoA-ACP transacilase (FabD) para formar malonil-ACP. A biossíntese de ácidos graxos propriamente dita é iniciada pela condensação de malonil-ACP e acetilCoA, catalisada pela $\beta$-cetoacil-ACP sintase III (FabH), resultando em $\beta$-cetobutiril-ACP e $\mathrm{CO}_{2}$. Logo após, ocorre série cíclica de etapas, que são repetidas até que determinado ácido graxo saturado de comprimento apropriado seja produzido. A primeira etapa de tal ciclo, catalisada pela $\beta$-cetoacil-ACP redutase (FabG), é a redução NADPH-dependente do grupo cetona por um grupo hidroxila. Em seguida, molécula de água é removida pela $\beta$-hidroxiacil-ACP desidratase (FabA ou FabZ), formando trans-2-enoil-ACP. A etapa final é a redução da dupla ligação do intermediário enoil pela NADH-dependente trans2-enoil-ACP redutase (FabI, FabK ou FabL) para formar acil-ACP. Ciclos subseqüentes são iniciados pela condensação do malonil-ACP com o acil-ACP, catalisada pela $\beta$-cetoacil-ACP sintase ou sintetase (FabB ou FabF) (Heath, White, Rock, 2001, 2002; Barry et al., 1998).

\section{Enoil-ACP reductase (ENR)}

A enzima enoil-ACP redutase (ENR) merece destaque, pois catalisa a reação principal do processo bioquímico de biossíntese de ácidos graxos e regula o ciclo de alongamento de ácidos graxos. A reação consiste na redução estereoespecífica, NADH-dependente, de ácidos graxos $\alpha, \beta$-insaturados ligados à proteína acil-carregadora (Pasqualoto, 2003; Barry et al., 1998).

A maioria das bactérias possui o gene $f a b I$, facilmente identificado em seus cromossomos, e expressa proteínas que são em torno de $40 \%$ idênticas no sítio ativo, contendo os resíduos catalíticos conservados Tyr156 e Lys 163 (Tabela I) (Heath, White, Rock, 2002).

A enzima homóloga da FabI na micobactéria é denominada InhA. Tais enzimas são essencialmente idênticas considerando sua estrutura terciária, exceto pela presença de uma longa alça de interação com o substrato, encontrada na InhA, que pode acarretar diferenças de especificidade ao substrato (Heath, White, Rock, 2002).

$\mathrm{O}$ conhecimento das características bioquímicas e estruturais das enzimas ENR supre informações importantes de como as mutações interferem nas interações fármaco-receptor e permite o planejamento de novos agentes terapêuticos eficientes contra as cepas sensíveis e MDR (Pasqualoto, 2003).

TABELA I - Expressão das isoformas de ENR em diferentes microrganismos

\begin{tabular}{lccc}
\hline Microrganismo & \multicolumn{3}{c}{ Expressão das isoformas de ENR } \\
& FabI & FabK & FabL \\
\hline Escherichia coli & Sim & Não & Não \\
Streptococcus pneumoniae & Não & Sim & Não \\
Bacillus subtilis & Sim & Não & Sim \\
Pseudomonas aeruginosa & Sim & Prevista & Não \\
Staphylococcus aureus & Sim & Não & Não \\
Mycobacterium tuberculosis & Sim & Prevista & Não \\
Plasmodium falciparum & Sim & Não & Não \\
\hline
\end{tabular}

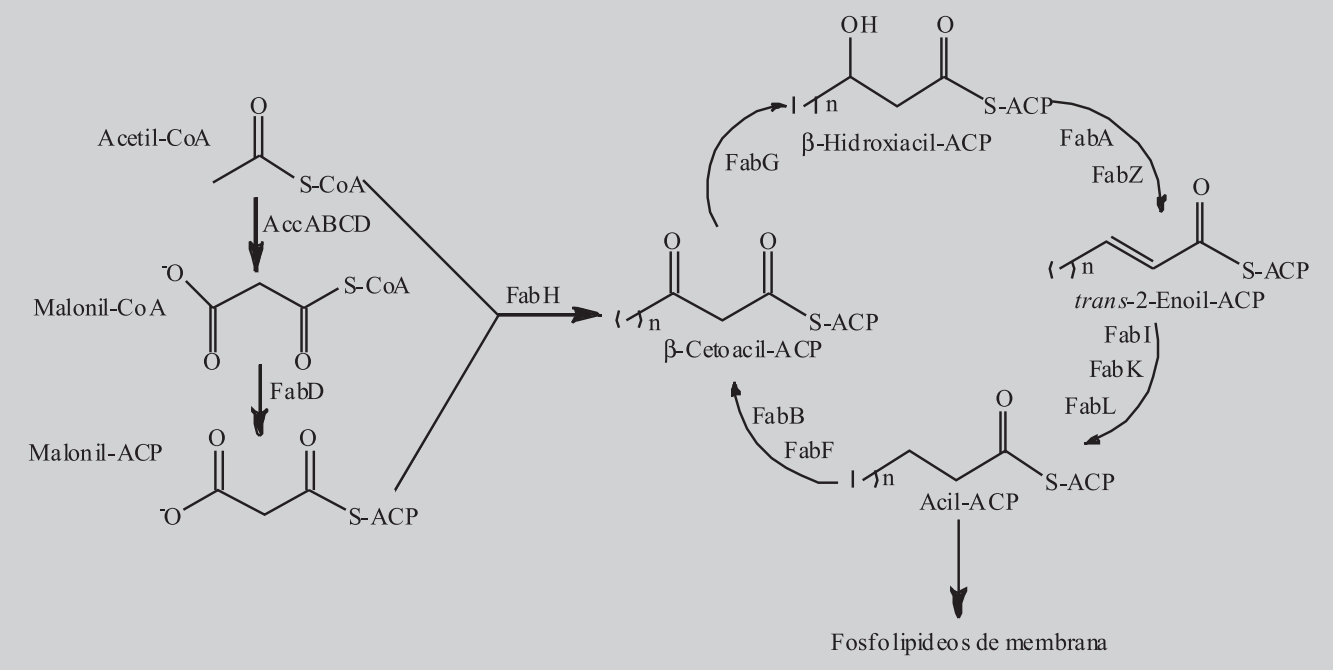

FIGURA 2 - Sistema FAS II de biossíntese de ácidos graxos. 


\section{INIBIDORES DE ENR}

Três classes de inibidores de ENR complexados com seus respectivos alvos enzimáticos foram caracterizados: isoniazida, que inibe a InhA, ENR do M. tuberculosis (Rozwarski et al., 1998), diazoborinas (Baldock et al., 1998) e triclosana (Stewart et al., 1999), compostos que inibem a FabI, ENR da E. coli (Figura 3).

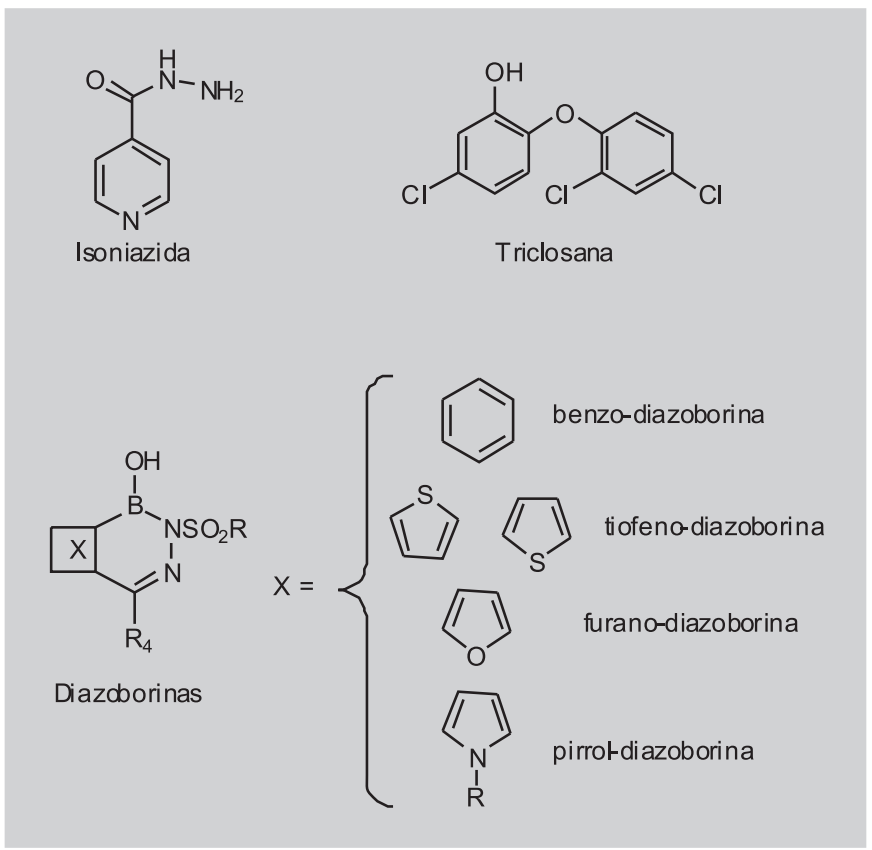

FIGURA 3 - Estruturas químicas de alguns inibidores de ENR.

\section{Isoniazida (INH)}

A atividade antimicobacteriana da isoniazida foi descoberta em 1952 e ainda hoje a INH é considerada como fármaco-base da quimioterapia anti-TB, tanto para infecções ativas quanto para aquelas latentes. Isso é devido à excelente atividade, ao baixo custo e à relativamente baixa toxicidade deste fármaco (Vilchèze et al., 2006). Logo após a introdução da INH na terapêutica foram isoladas cepas resistentes (Middlebrook, Cohn, 1953). Desde a sua introdução na terapêutica, centenas de derivados têm sido sintetizados e testados. Todavia, praticamente nenhum deles apresentou atividade antimicobacteriana superior à do fármaco (Scior, Garcés-Eisele, 2006).

Apesar da estrutura química simples, o mecanismo de ação da INH contra $o$ M. tuberculosis é complexo. A utilização de ferramentas genéticas se fez necessária ao estudo completo de possíveis mecanismos de ação da INH (Vilchèze, Jacobs, 2007).Vários estudos bioquímicos e genéticos têm confirmado o alvo primário da INH como sendo a proteína codificada pelo gene inha (Banerjee et al., 1994; Quémard et al., 1995; Vilchèze et al., 2000; Larsen et al., 2002; Kremer et al., 2003). Recentemente, a transferência de um ponto de mutação no gene inha do $M$. tuberculosis confirmou o alvo de ação da INH (Vilchèze et al., 2006).

A INH é um pró-fármaco (bioprecursor), que é ativado in vivo pela enzima KatG, uma catalase-peroxidase micobacteriana (Zhang et al., 1992; Rozwaski et al., 1999), para gerar a forma ativa (ânion ou radical), que se liga covalentemente ao átomo de carbono na posição 4 do anel nicotinamida do cofator (NAD, nicotinamida adenina dinucleotídeo), formando um aduto no sítio ativo da enzima InhA, inativando-a (Figura 4). Em conseqüência, há a interrupção da biossíntese dos ácidos micólicos, principais constituintes da parede celular micobacteriana (Rozwaski et al., 1998).

$\mathrm{Na}$ estrutura do complexo InhA-NAD-INH, obtido por cristalografia de raios-X (código de entrada do PDB 1zid) (Rozwaski et al., 1998), a orientação do grupo isonicotínico-acil em relação ao cofator NAD é de tal forma que o oxigênio da carbonila fica posicionado entre dois doadores de ligação hidrogênio, o nitrogênio da amida do anel nicotinamida e o oxigênio da 2'-hidroxila do anel da ribose. Em adição, o átomo de nitrogênio do grupo isonicotínico-acil participa de ligação hidrogênio com uma molécula de água presente no sítio ativo, que interage com a cadeia lateral do resíduo Met155. O anel piridina do grupo isonicotínico-acil está circundado por resíduos hidrofóbicos, que incluem os aminoácidos Phe149, Gly192, Pro193, Leu218, Tyr158 e Trp222. Em adição, a cadeia lateral do resíduo Phe149 participa de interação de transferência de carga do tipo $\pi$-stacking com o anel aromático do inibidor (Figura 5). (Rozwaski et al., 1998).

As mutações enzimáticas próximas ao sítio ativo, resultantes da substituição de um único aminoácido, como por exemplo, serina por alanina na posição 94 [Ser94Ala] (Dessen et al., 1995; Quémard et al., 1995; Baldock et al., 1996; Rozwarski, et al., 1998) são suficientes para conferir resistência à INH. Adicionalmente, mutações resultantes da substituição dos resíduos Ser94, já mencionados, Ile194, Ile21, Ile95 e Val78 também conferem resistência à INH (Banerjee et al., 1998; Morlock et al., 2003; Kruh et al., 2007; Dias et al., 2007).

Cabe ressaltar que apesar das mutações no gene inha serem conhecidas por facilitar a resistência à INH, a InhA permanece sendo um bom alvo para o planejamento de fármacos potencialmente tuberculostáticos. As razões para tal afirmativa são as seguintes: (i) a maioria das mutações encontradas em isolados clínicos resistentes à INH são associadas à enzima ativadora da $\mathrm{INH}$, a KatG; (ii) somente uma 


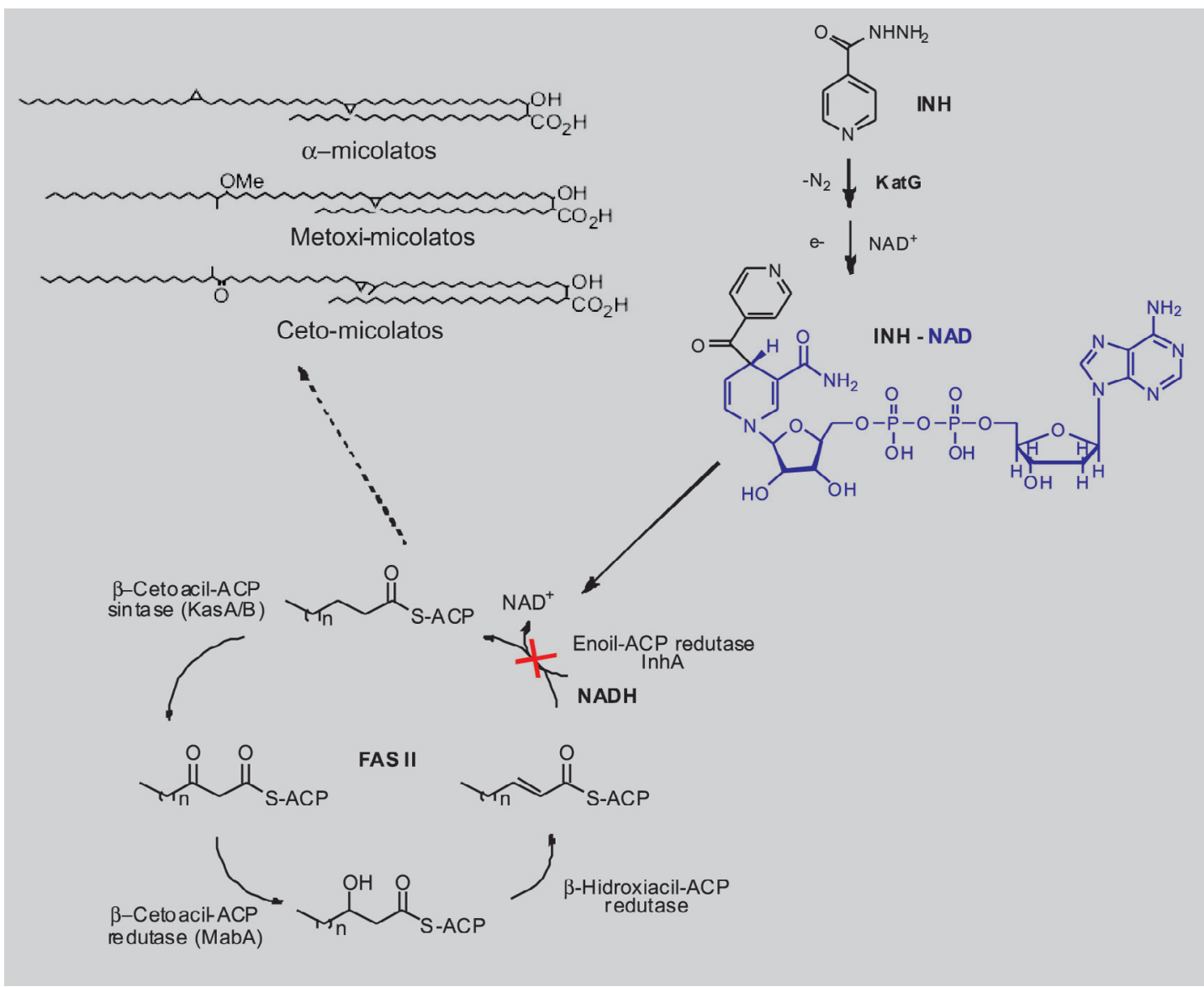

FIGURA 4 - Principal mecanismo de ação da INH. O fármaco INH é ativado pela KatG para formar o aduto INH-NAD. $\mathrm{O}$ aduto inibe a InhA e, assim, a biossíntese de ácidos graxos (FAS II), que sintetiza os ácidos micólicos (representação de três classes de ácidos micólicos: $\alpha$-micolatos, metóxi-micolatos e ceto-micolatos), é interrompida provocando a morte celular. (Vilchèze, Jacobs, 2007).

enoil-acp redutase é encontrada no $M$. tuberculosis; (iii) a InhA é uma enzima específica da micobactéria, não interferindo no sistema FAS I humano (Rozwarski et al., 1999).

Considerando que o mecanismo predominante de resistência à INH deve-se à mutações na KatG (Heym et al., 1994; Musser et al., 1996; Ramaswam et al.; 2003, agentes que inibem a InhA, mas que não requerem ativação pela KatG, são promissores como novos protótipos de atividade contra cepas MDR de M. tuberculosis (Rawat, Whitty, Tonge, 2003; Sullivan et al., 2006).

Portanto, o aduto isonicotínico-acil:NAD pode ser utilizado como protótipo ao planejamento de agentes tuberculostáticos que inibem a InhA, sem necessitar de ativação pela KatG (Pasqualoto, Ferreira, 2001).

\section{Diazoborinas (DZB)}

Diazoborinas representam uma família experimental de compostos antibacterianos, que possuem um anel heterocíclico 1,2-diazino contendo boro como elemento estrutural importante (Figura 3). O principal problema desta classe de compostos é o seu inerente potencial tóxico, o qual é provavelmente devido à porção amida ácida arenoborônica da molécula (Grassberger et al., 1984; Baldock et al., 1998).

Grassberger e colaboradores (1984) foram os primeiros a descrever a síntese de DZB e avaliaram sua atividade biológica in vitro e in vivo. A partir de tal estudo, discutiram-se algumas relações entre estrutura química e atividade biológica.

As DZB apresentaram atividade in vitro contra várias espécies de bactérias Gram-negativas e contra o $M$. tuberculosis. Em geral, as tiofeno-diazoborinas foram os inibidores mais potentes, seguidos pelas benzodiazoborinas e furano-diazoborinas, enquanto que as pirrol-diazoborinas foram totalmente inativas. Derivados sem o átomo de boro possuem pouca ou nenhuma atividade (Baldock et al., 1998). 


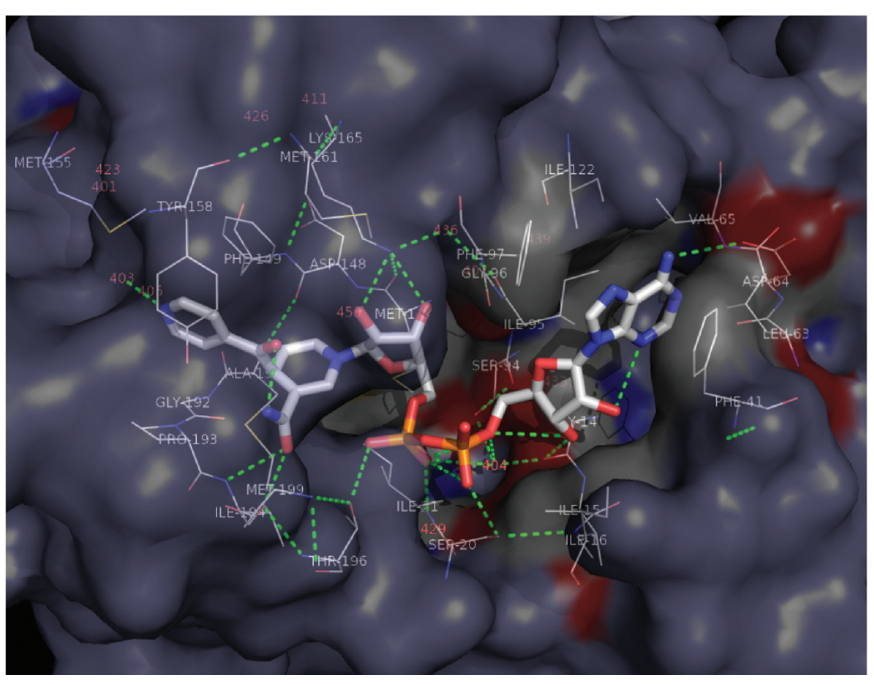

FIGURA 5 - Representação do aduto INH-NAD no sítio ativo da InhA (1zid) (programa PyMOL viewer). O aduto INH-NAD está apresentado em modelo palito. Os átomos de hidrogênio não estão representados. Os átomos de carbono estão em branco, nitrogênios em azul, oxigênios em vermelho e fósforo em laranja. Os resíduos de aminoácido do sítio ativo estão destacados: Phe149, Gly192, Pro193, Leu218, Tyr158, Trp222, Met155, Ile194, Ser94, Ala22, Gly14, Ser20, Thr17, Leu197, Ile21, Ile25, Thr196 e Lys 165. As moléculas de água que participam de interações importantes estão enumeradas em vermelho. As interações do tipo ligação hidrogênio estão tracejadas em verde.

A necessidade da presença do boro à atividade biológica se esclarece através da análise da estrutura cristalográfica do complexo FabI-NAD-tiofenodiazoborina e FabI-NAD-benzodiazoborina (códigos de entrada do PDB $1 d f h$ e $1 d f h$, respectivamente). O boro forma ligação covalente com a 2'-hidroxila da ribose próxima ao anel nicotinamida do cofator NAD no sítio ativo da enzima, sendo, portanto, essencial à atividade antibacteriana (Levy et al., 2001; Baldock et al., 1998).

\section{Triclosana (TCL)}

A triclosana é um bisfenol clorado (Figura 3), originalmente patenteada como herbicida, mas logo descobriuse seu amplo espectro antibacteriano (Heath et al., 1998). O grupo hidroxila é essencial à atividade biológica e a troca da ponte de $O$ por $S$ diminui drasticamente a atividade (Heath et al., 1998). Devido ao seu perfil de segurança favorável, a TCLé amplamente utilizada como conservante em produtos de higiene pessoal, como sabonetes e pastas de dentes (Bhargava, Leonard, 1996).

A primeira indicação de que a TCL inibia a biossíntese de ácidos graxos foi descrita por McMurry e colaboradores (1998). Estes pesquisadores isolaram cepas de E.coli resistentes à TCL e verificaram que a resistência residia no gene $f a b I$. Evidências diretas da FabI como alvo bioquímico da TCL foram obtidas do isolamento de mutantes naturais resistentes e da demonstração de resistência da proteína mutante purificada à TCL, em contraste ao observado com a enzima selvagem, que era sensível ao fármaco (Bhargava, Leonard, 1996).

Estudos mais recentes constataram, definitivamente, que tal etapa da biossíntese de ácidos graxos é o único alvo da TCL (Heath, Rock, 2000; Heath et al., 2000).

Ao contrário das DZB e da INH, a TCL não se liga de forma covalente ao cofator NAD no sítio ativo da ENR, FabI. Na estrutura do complexo FabI-NAD-TCL, obtida por cristalografia de raios-X (código de entrada do PDB $1 d 8 a$ ), observa-se que o anel hidroxi-cloro-fenil da TCLé coplanar ao anel nicotinamida do NAD, facilitando interações de transferência de carga do tipo $\pi$ stacking e, também, interações com as cadeias laterais dos resíduos Tyr156 e Tyr146, do sítio ativo. O grupo 2'-hidroxila da primeira ribose do NAD e a hidroxila do resíduo Tyr156 participam de ligação hidrogênio com a hidroxila da TCL. O cloro, na posição 4 do anel fenila da TCL, é aceptor de ligação hidrogênio da amida do resíduo Ala95 e participa de interações hidrofóbicas com a cadeia lateral do resíduo de aminoácido Met159 (Heath, White, Rock, 2001).

A TCL também inibe a InhA, de maneira similar à FabI (Parikh, Xiao, Tonge, 2000). Ensaios com cepas resistentes à INH mostraram que existe resistência cruzada com a TCL (Mcmurry, Oethinger, Levy, 1998).

\section{PLANEJAMENTO DE NOVOS INIBIDORES DE ENR}

Os inibidores de ENR - isoniazida, triclosana e diazoborinas - apresentam mecanismo de ação similar. Cada um se liga ao sítio ativo da ENR e forma um complexo estável com o cofator NAD. Enquanto a TCL e as DZB interagem com o grupo 2'-hidroxila da ribose do cofator, a INH forma um complexo covalente com o anel nicotinamida. Além disso, a INH ocupa posição diferente no sítio ativo, quando comparada aos outros inibidores descritos. Tal diferença pode ser explorada no planejamento de novos inibidores que ocupem ambas as regiões no sítio ativo da enzima (Heath, White, Rock, 2001).

Em adição, devido às estruturas químicas simples da TCL e da INH e ao perfil de toxicidade favorável, análogos destes fármacos podem ser planejados visando melhorar a atividade biológica, principalmente para cepas resistentes (Oliveira et al., 2007). 
A abordagem racional no planejamento de novos fármacos tem como uma de suas premissas a descoberta de alvos seletivos para a ação de novos agentes terapêuticos e pode buscar subsídios na aplicação de técnicas computacionais (CADD - Computer-Assisted Drug Design - planejamento de fármacos auxiliado pelo computador), para as quais a modelagem molecular é ferramenta importante (Patrick, 2005).

\section{Compostos em desenvolvimento}

Apesar dos avanços tecnológicos, a informação-chave que permite a abordagem racional ao planejamento de fármacos é o conhecimento da etiologia de determinada doença, ou pelo menos, dos processos bioquímicos nela envolvidos (Cohen, 1996).

Com a proposta de contribuir para o planejamento racional de novos protótipos tuberculostáticos, Pasqualoto e colaboradores (2004) realizaram estudo baseado no mecanismo de ação, empregando análise quantitativa na quarta dimensão entre estrutura química e atividade biológica, independente do receptor (QSAR 4D-IR) (Hopfinger et al., 1997), em um conjunto de 37 hidrazidas, análogas à INH. Os resultados indicaram que grupos apolares na porção acil dos ligantes (adutos) diminuem, significativamente, a atividade biológica. Modificações moleculares na porção NAD do ligante (aduto), incluindo grupos apolares, doadores e aceptores de ligação hidrogênio, aparentemente, melhoram as interações do ligante com os resíduos de aminoácidos Met161, Pro193, Ile194, Thr196, Leu197, Thr17 e Ser20 do sítio ativo da InhA (Pasqualoto et al., 2004).

Sullivan e colaboradores (2006) utilizaram o planejamento baseado na estrutura (Structure-Based Drug Design, SBDD), para explorar as interações de análogos da TCL. Um comjunto de éteres difenilalquílicos foi desenvolvido. Tais compostos são potentes inibidores in vitro da InhA e previnem o crescimento de ambas as cepas de $M$. tuberculosis, sensíveis e resistentes à INH (composto 1, Figura 6). Além disso, compostos baseados em éteres difenílicos, inibidores da InhA, não requerem ativação da KatG e, por isso, evitam o mecanismo normal de resistência da INH.

O advento da robótica e a miniaturização dos testes in vitro em células geneticamente modificadas contribuíram para o desenvolvimento dos ensaios biológicos automatizados em larga escala (High Throughput Screening, HTS) (Macarron, 2006). Como a síntese combinatória produz grande quantidade de compostos em curto espaço de tempo, então os testes biológicos devem ser rápidos e automatizados para acompanhar o processo (Patrick, 2005).
Já a triagem virtual (Virtual Screening, VS) é uma estratégia in silico bastante difundida que emprega os métodos de docagem molecular. Esta estratégia envolve a análise de grandes bases virtuais de compostos através de métodos computacionais com o objetivo de identificar um pequeno subgrupo de compostos que podem ser adquiridos através de representantes comerciais ou de síntese orgânica para avaliação da atividade biológica (Klebe, 2006). Portanto, a triagem virtual permite a seleção de milhares de compostos, que são submetidos a grande número de testes biológicos automatizados (Wermuth, 2003).

Kuo e colaboradores (2003) utilizaram as metodologias VS e HTS para identificar duas novas classes de inibidores da InhA, considerando piperazinas e pirazóis substituídos. Os compostos mais potentes apresentaram valores de $\mathrm{IC}_{50}$ de $0,16 \mu \mathrm{M}$ (composto 2, Figura 6) e 2,4 $\mu \mathrm{M}$ (composto 3, Figura 6) para inibição da InhA. Além disso, estes inibidores também foram ativos contra cepas de $P$. falciparum multi-resistentes (Kuo et al., 2003).

Payne e colaboradores (2002) descobriram e caracterizaram uma nova classe de antibacterianos dirigidos à FabI também utilizando o VS. Foram utilizados 305.000 compostos da coleção da GlaxoSmithKline. A otimização dos compostos permitiu a identificação de um derivado lactâmico (composto 4, Figura 6), que apresentou aumento de potência em 100 vezes contra a FabI do $S$. aureus.

He e colaboradores (2006), também aplicando a metodologia de HTS, descobriram série de pirrolidina carboxamidas como nova classe de potentes inibidores da InhA. A potência do composto protótipo foi aumentada em mais de 160 vezes por otimizações subseqüentes, e seu valor de $\mathrm{IC}_{50}$ chegou a $0,14 \mu \mathrm{M}$ (composto 5, Figura 6).

$\mathrm{Na}$ tentativa de contribuir para o planejamento racional de novos protótipos antimicobacterianos, estamos sintetizando um conjunto de 27 hidrazidas, selecionadas da literatura (Bernstein et al., 1952, 1953a, b; Yale et al., 1953) e com mecanismo de ação possivelmente comum ao da INH, para realizar ensaios de inibição enzimática, $K i$, na enzima enoil-acp redutase do $M$. tuberculosis, InhA, de acordo com metodologia descrita por Vilchèze e colaboradores (2005). Tais ensaios enzimáticos serão realizados em colaboração com o grupo do Prof. James Sacchettini, na Texas A\&M University, EUA.

A partir da obtenção dos dados de atividade biológica quantitativos, serão aplicadas as seguintes metodologias do CADD: QSAR-3D em campo de força de energia livre (Free-Energy Force Field, FEFF 3D-QSAR) (Tokarski, Hopfinger, 1997a, b) no qual os dados de $K i$ serão utilizados para estimar a energia livre de interação $(\Delta \mathrm{G})$, e QSAR-4D dependente do receptor, (DR) (Pan, Tseng, Hopfinger, 2003; 


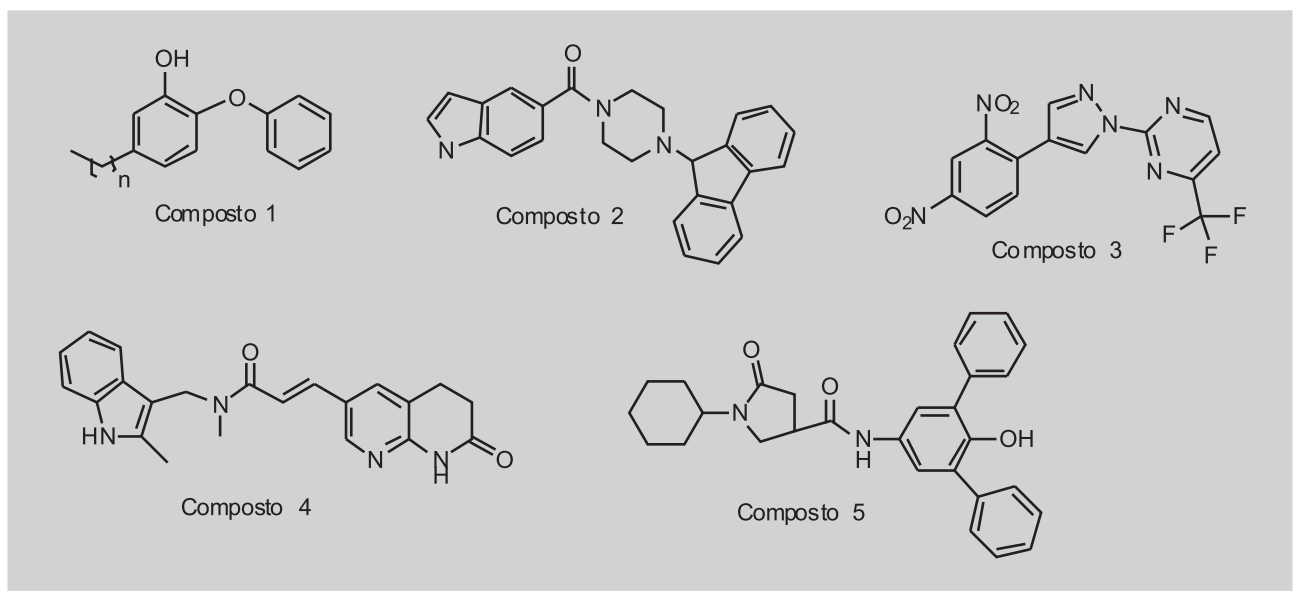

FIGURA 6 - Inibidores de ENR descobertos recentemente.

Pan et al., 2004). Os modelos gerados nas abordagens do CADD permitirão determinar requisitos estruturais relevantes à atividade biológica do conjunto de hidrazidas investigado, que auxiliarão na previsão de atividade de novas moléculas e no planejamento racional de novos agentes antimicobacterianos.

\section{CONSIDERAÇÕES FINAIS}

A gravidade de muitas infecções bacterianas, com destaque para aquelas provocadas por micobactérias, caso da TB, conduz à necessidade de novas alternativas quimioterápicas para o seu controle. A busca urgente por novos agentes quimioterápicos é enfatizada, quando se considera o aparecimento de cepas resistentes do microrganismo.

O planejamento de fármacos mais eficazes e seguros tem sido o principal objetivo a ser alcançado no desenvolvimento de novas gerações de quimioterápicos. Neste sentido, enzimas presentes apenas na micobactéria, estão sendo estudadas e suas estruturas elucidadas.

O processo bacteriano/micobacteriano de biossíntese de ácidos graxos merece atenção especial, devido às diferenças bioquímicas e estruturais com relação ao mesmo processo no homem. Portanto, as enzimas que constituem tal processo bioquímico mostram-se promissoras como alvos para o planejamento de novos antibacterianos/ antimicobacterianos.

\section{ABSTRACT}

\section{Rational approach in the new antituberculosis agent design: inhibitors of $\mathrm{Inh} A$, the enoyl-ACP reductase from Mycobacterium tuberculosis}

tuberculosis (TB) has been among the worldwide health threats. Mycobacteria resistance to the drugs currently used in the therapeutics is the main cause of $T B$ resurgence. In view of this severe situation, the new and selective anti-TB design is of utmost importance. Fatty acid biosynthesis is a prokariontes and eucariontes biochemical process that supplies essential precursors for the assembly of important cellular components, such as phospholipids, lipoproteins, lipopolysaccharides, mycolic acids and cellular envelope. However, the biochemical and functional differences between the bacterial and mammals' fatty acid synthetic pathway have endowed the mycobacterial enzymes with distinct properties. These provide valuable opportunities for structure- or catalytic mechanism-based design of selective inhibitors as novel anti-TB drugs with improved properties. The enoylreductases are essential enzymes in the fatty acids elongation pathway towards the mycolic acids, the main mycobacteria cell wall constituents, biosynthesis and so they are potential targets to the rational new antimycobacteria drug design. This paper highlights recent approaches regarding the design of new anti-TB agents, particularly, the enoyl-ACP reductase inhibitors.

UNITERMS: Tuberculosis. Fatty acid/ biosynthesis. Mycolic acids. Mycobacteria drug resistance. Drugs/ Structure-based design. Enoyl-Act reductase/ inhibitors.

\section{AGRADECIMENTOS}

Os autores agradecem à FAPESP, à CAPES e ao CNPq pelo apoio financeiro. 


\section{REFERÊNCIAS BIBLIOGRÁFICAS}

BALDOCK, C.; de BOER, G.J.; RAFFERTY, J.B.; STUITJE, A.R.; RICE, D.W. Mechanism of action of diazaborines. Biochem. Pharmacol., v.55, p.1541-1549, 1998.

BALDOCK, C.; RAFFERTY, J.B.; SEDELNIKOVA, S.E.; BAKER, P.J.; STUITJE, A.R.; SLABAS, A.R.; HAWKES, T.R.; RICE, D.W. A mechanism of drug action revealed by structural studies of enoyl reductase. Science, v.274, p.2107-2110, 1996.

BANERJEE, A.; DUBNAU, E.; QUÉMARD, A.; BALASUBRAMANIAN, V.; UM, D.S.; WILSON, T. $\operatorname{inh} A$, a gene encoding a target for isoniazid and ethionamide in Mycobacterium tuberculosis. Science, v.263, p.227-230, 1994.

BANERJEE, A.; SUGANTINO, M.; SACCHETTINI, J.C.; JACOBS, W.R.Jr. The mabA gene from the inh $A$ operon of Mycobacterium tuberculosis encodes a 3-ketoacyl reductase that fails to confer isoniazid resistance. Microbiology, v.144, p.2697-2704, 1998.

BARRY III, C.E.; MDLULI, K. Drug sensitivity and environmental adaptation of mycobacterial cell wall components. Trends Microbiol., v.4, p.275-281, 1996.

BARRY III, C.E.; LEE, R.E.; MDLULI, K.; SAMPSON, A.E.; SCHROEDER, B.G.; SLAYDEN, R.A.; YUAN, Y. Mycolic acids: structure, biosynthesis and physiological functions. Prog. Lipid. Res., v.37, p.143-179, 1998.

BERNSTEIN, J.; LOTT, W.A.; STEINBERG, B.A.; YALE, H.L. Chemotherapy of experimental tuberculosis V.Isonicotinic acid hydrazide (nydrazid) and related compounds. Am. Rev. Tuberc., v.65, p.357-364, 1952.

BERNSTEIN, J.; JAMBOR, W.P.; LOTT, W.A.; PANSY, F.; STEINBERG, B.A.; YALE, H.L. Chemotherapy of experimental tuberculosis - VI. Derivatives of isoniazid. Am. Rev.Tuberc., v.67, p.354-365, 1953a.

BERNSTEIN, J.; JAMBOR, W.P.; LOTT, W.A.; PANSY, F.; STEINBERG, B.A.; YALE, H.L. Chemotherapy of experimental tuberculosis - VII. Heterocyclic acid hydrazides and derivatives. Am. Rev. Tuberc., v.67, p.366$375,1953 b$.
BHARGAVA, H.N.; LEONARD, P.A. Triclosan: applications and safety. Am. J. Infect. Control. v.24, p.209-218, 1996.

BRENAN, P.J.; NIKAIDO, H. The envelope of mycobacteria. Annu. Rev. Biochem., v.64, p.29-63, 1995.

COHEN, N.C. Guidebook on molecular modeling in drug design.San Diego: Academic Press, 1996. 361p.

CORBETT, E.L.; WATT, C.J.; WALKER, N.; MAHER, D.; WILLIAMS, B.G.; RAVIGLIONE. M.C.; DYE C. The growing burden of tuberculosis: global trends and interactions with the HIV epidemic. Arch. Intern. Med., v.163, n.9, p.1009-1212, 2003.

DESSEN, A.; QUÉMARD, A.; BLANCHARD, J.S.; Jr JACOBS, W.R.; SACCHETTINI, J.C. Crystal structure and fuction of the isoniazid target of Mycobacterium tuberculosis. Science, v.267, p.1638-1641, 1995.

Dias, M.V.B.; Vasconcelos, I.B.; Prado, A.M.X.; Fadel, V.; Basso, L.A.; DE AZEVEDO, W.F.; SANTOS, D.S. Crystallographic studies on the binding of isonicotinylNAD adduct to wild-type and isoniazid resistant 2-transenoyl-ACP (CoA) reductase from Mycobacterium tuberculosis. J. Struct. Biol., v.159, p.369-380, 2007.

DOLIN, P.J.; RAVIGLIONE, M.C.; KOCHI, A. Global tuberculosis incidence and mortality during 1990-2000. Bull. WHO, v.72, p.213-220, 1994.

ESPINAL, M.A. The global situation of MDR-TB. Tuberculosis, v.83, p.44-51, 2003.

GANDY, M.; ZUMLA, A. The resurgence of disease: social and historical perspectives on the 'new' tuberculosis. Soc. Sci. Med., v.55, p.385-396, 2002.

GRASSBERGER, M.A.; TURNOWSKY, F.; HILDEBRANDT, J. Preparation and antibacterial activities of new 1,2,3-diazaborine derivatives and analogues. J. Med. Chem., v.27, p.947-953, 1984.

HE, X.; ALIAN, A.; STROUD, R.; MONTELLANO, P.R.O. Pyrrolidine carboxamides as a novel class of inhibitors of enoyl acyl carrier protein reductase from Mycobacterium tuberculosis. J. Med. Chem., v.49, p.6308-6323, 2006. 
HEATH, R.J.; LI, J.; ROLAND, G.E.; ROCK, C.O. Inhibition of the Staphylococcus aureus NADPHdependent enoyl-acyl carrier protein reductase by triclosan and hexachlorophene. J. Biol. Chem., v.275, p.46544659, 2000.

HEATH, R.J.; ROCK, C.O. A triclosan-resistant bacterial enzyme. Nature, v.406, p.145-146, 2000.

HEATH, R.J.; WHITE, S.W.; ROCK, C.O. Lipid biosynthesis as a target for antibacterial agents. Prog. Lipid. Res., v.40, p.467-497, 2001.

HEATH, R.J.; WHITE, S.W.; ROCK, C.O. Inhibitors of fatty acid synthesis as antimicrobial chemotherapeutics. Appl. Microbiol. Biotechnol., v.58, p.695-703, 2002.

HEATH, R.J.; YU, Y-T; SHAPIRO, M.A.; OLSON, E.; ROCK, C.O. Broad spectrum antimicrobial biocides target the FabI component of fatty acid biosynthesis. $J$. Biol. Chem., v.273, p.30316-30321, 1998.

HEYM, B.; HONORE, N.; TRUFFOT-PERNOT, C.; BANERJEE, A.; SCHURRA, C.; JACOBS, W.R.; VAN EMBDEN, J.D.; GROSSET, J.H.; COLE, S. T. Implications of multidrug resistance for the future of shortcourse chemotherapy of tuberculosis: a molecular study. Lancet, v.344, p.293-298, 1994.

HOPFINGER, A. J.; WANG, S.; TOKARSKI, J. S.; JIN, B.; ALBUQUERQUE, M.; MADHAV, P.J.; DURAISWAMI, C. Construction of 3D-QSAR models using the 4D-QSAR analysis formalism. J. Am. Chem. Soc., v.119, p.10509-10524, 1997.

JANIN, Y.L. Antituberculosis drugs: ten years of research. Bioorg. Med. Chem., v.15, p.2479-2513, 2007.

JARLIER, V.; NIKAIDO, H. Mycobacterial cell wall: structure and role in natural resistance to antibiotics. FEMS Microbiol. Lett., v.15, p.11-18, 1994.

KLEBE, G. Virtual ligand screening: strategies, perspectives and limitations. Drug Discov.Today., v.11, p.580-594, 2006.
KREMER, L.; DOVER, L.G.; MORBIDONI, H.R.; VILCHÈZE, C.; MAUGHAN, W.N.; BAULARD, A.; TU, S.C.; HONORÉ, N.; DERETIC, V.; SACCHETTINI, J.C.; LOCHT, C.; JACOBS, W.R. Jr.; BESRA, G.S. Inhibition of InhA activity, but not KasA activity, induces formation of a KasA-containing complex in mycobacteria. J. Biol. Chem., v.278, n.23, p.2054720554, 2003.

KRUH, N.A.; RAWAT, R.; RUZSICSKA, B.P.; TONGE, P.J. Probing mechanisms of resistance to the tuberculosis drug isoniazid: Conformational changes caused by inhibition of InhA, the enoyl reductase from Mycobacterium tuberculosis. Protein Sci., v.16, p.1617$1627,2007$.

KUO, M.R.; MORBIDONI, H.R.; ALLAND, D.; SNEDDON, S.F.; GOURLIE, B.B.; STAVESKI, M.M.; LEONARD, M.; GREGORY, J.S.; JANJIGIAN, A.D.; YEE, C.; MUSSER, J.M.; KREISWIRTH, B.; IWAMOTO, H.; PEROZZO, R.; JACOBS, W.R.Jr.; SACCHETTINI, J.C.; FIDOCK, D.A. Targeting tuberculosis and malaria through inhibition of enoyl reductase: compound activity and structural data. J. Biol. Chem., v.278, p.20851-20859, 2003.

LARSEN, M.H.; VILCHÈZE, C.; KREMER, L.; BESRA, G.S.; PARSONS, L.; SALFINGER, M.; HEIFETS, L.; HAZBON, M.H.; ALLAND, D.; SACCHETTINI, J.C.; JACOBS, W.R.Jr. Overexpression of inhA, but not kasA, confers resistance to isoniazid and ethionamide in Mycobacterium smegmatis, $M$. bovis $B C G$ and $M$. tuberculosis. Mol. Microbiol., v.46, p.453-466, 2002.

LEVY, C.W.; BALDOCK, C.; WALLACE, A.J.; SEDELNIKOVA, S.; VINER, R.C.; CLOUGH, J.M.; STUITJE, A.R.; SLABAS, A.R.; RICE, D.W.; RAFFERTY, J.B. A study of the structure-activity relationship for diazaborine inhibition of Escherichia coli enoyl-acp reductase. J. Mol. Biol., v.309, p.171-180, 2001.

MACARRON, R. Critical review of the role of HTS in drug discovery. Drug Discov. Today, v.11, p.277-279, 2006.

MARRAKCHI, H.; ZHANG, Y.M.; ROCK, C.O. Mechanistic diversity and regulation of Type II fatty acid synthesis. Biochem. Soc. Trans., v.30, p.1050-1055, 2002. 
McMURRY, L.M.; OETHINGER, M.; LEVY, S.B. Triclosan targets lipid synthesis. Nature, v.394, p.531532, 1998.

MIDDLEBROOK, G.; COHN, M.L. Some observations on the pathogenicity of isoniazid-resistant variants of tubercle bacilli. Science, v.118, p.297-299, 1953.

MORLOCK, G.P.; METCHOCK, B.; SIKES, D.; CRAWFORD, J.T.; COOKSEY, R.C. ethA, inhA, and $\mathrm{kat} G$ loci of ethionamide-resistant clinical Mycobacterium tuberculosis isolates. Antimicrob. Agents Chemother., v.47, p.3799-3805, 2003.

MUSSER, J.M.; KAPUR, V.; WILLIAMS, D.L.; KREISWIRTH, B.N.; van SOOLINGEN, D.; van EMBDEN, J.D. Characterization of the catalaseperoxidase gene (katG) and inhA locus in isoniazidresistant and -susceptible strains of Mycobacterium tuberculosis by automated DNA sequencing: restricted array of mutations associated with drug resistance. $J$. Infect. Dis., v.173, p.196-202, 1996.

NAYYAR, A.; JAIN, R. Recent advances in new structural classes of anti-tuberculosis agents. Curr. Med. Chem., v.12, n.16, p.1873-1886, 2005.

OLIVEIRA, J.S.; PEREIRA, J.H.; CANDURI, F.; RODRIGUES, N.C.; DE SOUZA, O.N.; DE AZEVEDO, W.F.Jr.; BASSO, L.A.; SANTOS, D.S. Crystallographic and pre-steady-state kinetics studies on binding of NADH to wild-type and isoniazid-resistant enoyl-ACP $(\mathrm{CoA})$ reductase enzymes from Mycobacterium tuberculosis. J. Mol. Biol., v.359, p.646666, 2006.

OLIVEIRA, J.S.; VASCONCELOS, I.B.; MOREIRA, I.S.; SANTOS, D.S.; BASSO, L.A. Enoyl reductases as targets for the development of anti-tubercular and antimalarial agents. Curr. Drug Targets, v.8, p.399-411, 2007.

PAN, D.; LIU, J.; SENESE, C.; HOPFINGER, A.J.; TSENG, Y. Characterization of a ligand-receptor binding event using receptor-dependent four-dimensional quantitative structure-activity relationship analysis. J. Med. Chem., v.47, p.3075-3088, 2004.
PAN, D.; TSENG, Y.; HOPFINGER, A.J. Quantitative structure -based design: Formalism and application of receptor-dependent RD-4D-QSAR analysis to a set of glucose analogue inhibitors of glycogen phosphorylase. $J$. Chem. Inf. Comput. Soc., v.43, p.1594-1607, 2003.

PARIKH, S.L.; XIAO, G.; TONGE, P.J. Inhibition of InhA, the enoyl reductase from Mycobacterium tuberculosis, by triclosan and isoniazid. Biochemistry, v.39, p.7645-7650, 2000 .

PASQUALOTO, K.F.; FERREIRA, E.I. An approach for the rational design of new antituberculosis agents. Curr. Drug Targets, v.2, p.427-437, 2001.

PASQUALOTO, K.F.M. Planejamento racional de tuberculostáticos com base na estrutura dos ácidos micólicos da parede celular do Mycobacterium tuberculosis. São Paulo, 2003.231p. [Tese de Doutorado. Faculdade de Ciências Farmacêuticas. Universidade de São Paulo].

PASQUALOTO, K.F.M.; FERREIRA, E.I.; SANTOS, O.A.F.; HOPFINGER, A.J. Rational design of new antituberculosis agents: Receptor-independent fourdimensional quantitative structure-activity relationship analysis of a set of isoniazid derivatives. J. Med. Chem., v.47, p.3755-3764, 2004.

PATRICK, G.L. An introduction to medicinal chemistry. 3.ed. Oxford: Oxford University Press, 2005. p.163-184.

PAYNE, D.J.; MILLER, W.H.; BERRY, V.; BROSKY, J.; BURGESS, W.J.; CHEN, E.; DEWOLF, W.E.; FOSBERRY, A.P.; GREENWOOD, R.; HEAD, M.S.; HEERDING, D.A.; JANSON, C.A.; JAWORSKI, D.D.; KELLER, P.M.; MANLEY, P.J.; MOORE, T.D.; NEWLANDER, K.A.; PEARSON, S.; POLIZZI, B.J.; QIU, X.; RITTENHOUSE, S.F.; RADOSTI, C.S.; SALYERS, K.L.; SEEFELD, M.A.; SMYTH, M.G.; TAKATA, D.T.; UZINSKAS, I.N.; VAIDYA, K.; WALLIS, N.G.; WINRAM, S.B.; YUAN, C.C.K.; HUFFMAN, W. Discovery of a novel and potent class of FabI-directed antibacterial agents. Antimicrob. Agents Chemother., v.46, p.3118-3124, 2002.

QUÉMARD, A.; SACCHETTINI, J.C.; DESSEN, A.; VILCHÈZE, C.; BITTMAN, R.; Jr. JACOBS, W.R.; BLANCHARD, J.S. Enzymatic characterization of the target for isoniazid in Mycobacterium tuberculosis. Biochemistry, v.34, p.8235-8241, 1995. 
RAMASWAMY, S.V.; REICH, R.; DOU, S.J.; JASPERSE, L.; PAN, X.; WANGER, A.; QUITUGUA, T.; GRAVISS, E.A. Single nucleotide polymorphisms in genes associated with isoniazid resistance in Mycobacterium tuberculosis. Antimicrob. Agents Chemother., v.47, p.1241-1250, 2003.

RAWAT, R.; WHITTY,A.; TONGE, P.J. The isoniazid-NAD adduct is a slow, tight-binding inhibitor of InhA, the Mycobacterium tuberculosis enoyl reductase: adduct affinity and drug resistance. Proc. Natl. Acad. Sci. U.S.A., v.100, n.24, p.13881-13886, 2003.

ROZWARSKI, D.A.; GRANT, G.A.; Jr. BARTON, D.H.R.; JACOBS, W.R.; SACCHETTINI, J.C. Modification of the NADH of the isoniazid target (InhA) from Mycobacterium tuberculosis. Science, v.279, p.98-102, 1998.

ROZWARSKI, D.A.; VILCHEZE, C.; SUGANTINO, M.; BITTMAN, R.; SACCHETTINI, J.C. Crystal structure of the Mycobacterium tuberculosis enoyl-acp reductase, InhA, in complex with $\mathrm{NAD}^{+}$and a $\mathrm{C} 16$ fatty acyl substrate. J. Biol. Chem., v.27, p.15582-15589, 1999.

SCIOR, T.; GARCES-EISELE, S.J. Isoniazid is not a lead compound for its pyridyl ring derivatives, isonicotinoyl amides, hydrazides, and hydrazones: a critical review. Curr. Med. Chem., v.13, p.2205-2219, 2006.

SINGH, S. Tuberculosis, focus on tropical diseases. Cur. Anaesthesia Crit. Care, v.15, p.165-171, 2004.

STEWART, M.J.; PARIKH, S.; XIAO, G.; TONGE, P.J.; KISKER, C. Structural basis and mechanism of enoyl reductase inhibition by triclosan. J. Mol. Biol., v.290, p.859-865, 1999.

SULLIVAN, T.J.; TRUGLIO, J.J.; BOYNE, M.E.; NOVICHENOK, P.; ZHANG, X.; STRATTON, C.F.; LI, H.J.; KAUR, T.; AMIN, A.; JOHNSON, F.; SLAYDEN, R.A.; KISKER, C.; TONGE, P.J. High affinity InhA inhibitors with activity against drug-resistant strains of Mycobacterium tuberculosis. ACS Chem. Biol., v.1, p.43-53, 2006.

TOKARSKI, J.S.; HOPFINGER, A.J. Constructing protein models for ligand-receptor binding thermodynamic simulations: An application to a set of peptidometic rennin inhibitors. J. Chem. Inf. Comput. Sci., v.37, p.779-791, 1997a.
TOKARSKI, J.S.; HOPFINGER, A.J. Prediction of ligandreceptor binding thermodynamics by free energy force field (FEFF) 3D-QSAR analysis: Application to a set of peptidometic rennin inhibitors. J. Chem. Inf. Comput. Sci., v.37, p.792-811, $1997 b$.

VILCHÈZE, C.; JACOBS, W.R.Jr. The mechanism of isoniazid killing: Clarity through the scope of genetics. Annu. Rev. Microbiol., v.61, p.35-50, 2007.

VILCHÈZE, C.; MORBIDONI, H.R.; WEISBROD, T.R.; IWAMOTO, H.; KUO, M.; SACCHETTINI, J.C.; JACOBS, W.R.Jr. Inactivation of the inhA-encoded fatty acid synthase II (FASII) enoyl-acyl carrier protein reductase induces accumulation of the FASI end products and cell lysis of Mycobacterium smegmatis. J. Bacteriol., v.182, p.4059-4067, 2000.

VILCHÈZE, C.; WANG, F.; ARAI, M.; HAZBON, M.H.; COLANGELI, R.; KREMER, L.; WEISBROD, T.R.; ALLAND, D.; SACCHETTINI, J.C.; JACOBS, W.R.Jr. Transfer of a point mutation in Mycobacterium tuberculosis inhA resolves the target of isoniazid. Nat. Med., v.12, p.1027-1029, 2006.

VILCHÈZE, C.; WEISBROD, T.R.; CHEN, B.; KREMER, L.; HAZBÓN, M.H.; WANG, F.; ALLAND D.; SACCHETTINI, J.C.; JACOBS, W.R.Jr. Altered $\mathrm{NADH} / \mathrm{NAD}^{+}$ratio mediates coresistance to isoniazid and ethionamide in Mycobacteria. Antimicrob. Agents Chemother., v.49, p.708-720, 2005.

WERMUTH, C.G. Strategies in the search for new lead compounds or original working hypothesis. In:_. The practice of medicinal chemistry. San Diego: Academic Press, 2003. p.69-87.

WHO. World Health Organization.Global tuberculosis control - surveillance, planning, financing. 2007. Disponível em: <http://www.who.int/tb/publications/ global_report/2007/en/index.html >. Acesso em: 12 out. 2007.

ZHANG, Y.; HEYM, B.; ALLEN, B.; YOUNG, D.; COLE, $\mathrm{S}$. The catalase-peroxidase gene and isoniazid resistance of Mycobacterium tuberculosis. Nature, v.358, p.591593, 1992.

ZHANG, Y. The magic bullets and tuberculosis drug targets. Annu. Rev. Pharmacol. Toxicol., v.45, p.529-564, 2005. 
ZHANG, Y.; POST-MARTENS, K.; DENKIN, S. New drug candidates and therapeutic targets for tuberculosis therapy. Drug. Discov. Today, v.11, p.21-27, 2006.
YALE, H.L.; LOSEE, K.; MARTINS, J.; HOLSING, M.; PERRY, F.M.; BERNSTEIN, J. Chemoterapy of experimental tuberculosis. VIII. The synthesis of acid hydrazides, their derivatives and related compounds. $J$. Am. Chem. Soc., v.75, p.1933-1942, 1953.

Recebido para publicação em 21 de janeiro de 2008 Aceito para publicação em 08 de maio de 2008 